\title{
Journal of Applied Mathematics and Mechanics
}

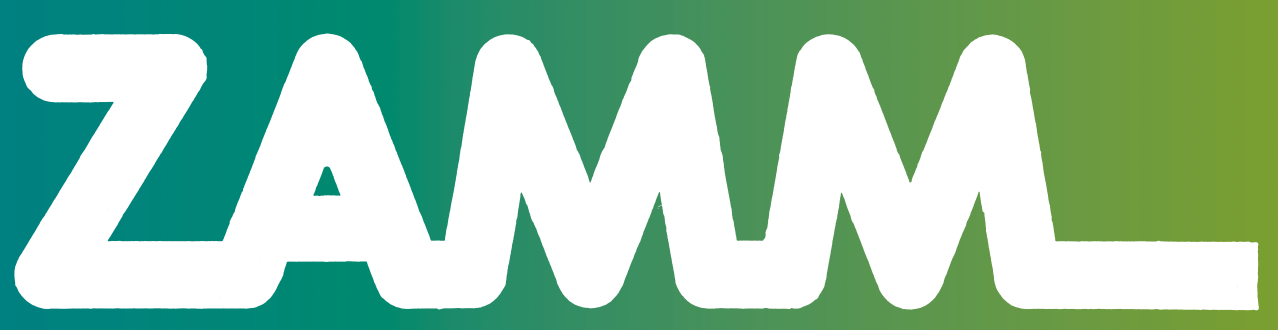

\section{Zeitschrift für Angewandte Mathematik und Mechanik Founded by Richard von Mises in 1921}

\section{Buckling and post-buckling of a nonlinearly elastic column}

\section{Brojan, A. Puksic, and F. Kosel}

Faculty of Mechanical Engineering, University of Ljubljana, Askerceva 6, 1000 Ljubljana, Slovenia

Received 25 January 2007, accepted 3 May 2007

Published online 25 July 2007

Key words stability, post-buckling, material nonlinearity, large deflections, critical force, Ludwick formula, bifurcation, limit load, finite disturbance buckling

The classical problem of buckling of an inextensible elastic column, under the action of a compressive force is examined. The column is made of nonlinearly elastic material for which the stress-strain relation is represented by the Ludwick constitutive law. An approximative formula for determination of the force at immediate post-buckling is given. Further post-buckling solutions are obtained for different values of the nonlinearity parameter by numerical integration using the Runge-Kutta-Fehlberg algorithm, and are presented in non-dimensional diagrams. It is shown that no bifurcation point is found in the case of nonlinearly elastic column.

Z. Angew. Math. Mech. 87, No. 7, 518-527 (2007) / DOI 10.1002/zamm.200710333 


\title{
Buckling and post-buckling of a nonlinearly elastic column
}

\author{
M. Brojan*, A. Puksic, and F. Kosel \\ Faculty of Mechanical Engineering, University of Ljubljana, Askerceva 6, 1000 Ljubljana, Slovenia
}

Received 25 January 2007, revised and accepted 3 May 2007

Published online 25 July 2007

\begin{abstract}
Key words stability, post-buckling, material nonlinearity, large deflections, critical force, Ludwick formula, bifurcation, limit load, finite disturbance buckling

The classical problem of buckling of an inextensible elastic column, under the action of a compressive force is examined. The column is made of nonlinearly elastic material for which the stress-strain relation is represented by the Ludwick constitutive law. An approximative formula for determination of the force at immediate post-buckling is given. Further post-buckling solutions are obtained for different values of the nonlinearity parameter by numerical integration using the Runge-Kutta-Fehlberg algorithm, and are presented in non-dimensional diagrams. It is shown that no bifurcation point is found in the case of nonlinearly elastic column.
\end{abstract}

\section{Introduction}

Buckling in particular occurs in a straight column that is subjected to an axial compressive load. If the compressive load is smaller than the critical load, the column is compressed but remains straight. On the other hand, if the compressive load exceeds the critical load, stability of the straight column can be lost and the column buckles into one of several stable curved states. This classical subject was first analyzed in the 18th century by Bernoulli and Euler. An exact analytical solution that governs the post-critical bending of columns (Euler's elastica) was obtained by Lagrange in terms of the Jacobi Elliptic functions. No matter how old this equilibrium problem is, it still attracts considerable attention of many scientists. An instructive historical overview and connection to the modern treatment of elastica problems was given by Komkov [1]. Kuznetsov and Levyakov [2,3] continued the work of Korobeinikov [4] and examined the stability, nonlinear deformation, and secondary loss of stability of simply supported and clamped columns. They found bifurcation points and solution branches that were not known previously. Similar results of the same topic were also obtained by Wu [5]. Wang [6] presented a thoughtful analysis for clamped-simply supported elastica applying a one-parameter shooting method for numerical solution as well as providing classical approximative techniques for initial post-buckling, with a perturbation approach, and an asymptotic expansion model for large loads. Another interesting study of buckling was the extension of Wang's work by Vaz and Silva [7]. They considered a rotational spring in lieu of the built-in end so that the solution may be tuned from the simply supported-clamped to the simply supported. A treatment of the buckling problem on the elastica of the column can also be found in [8-11].

In the recent years the importance of the subject of nonlinear elasticity is increasing due to an ever growing use of polymer and composite materials in many industrial applications. Much of the impetus for the theoretical developments in nonlinear elasticity came from the rubber industry because of the importance of natural and synthetic rubber in many engineering components. While understanding of nonlinear elasticity is in itself important, the theory also provides a gateway towards the understanding of more complex (non-elastic) material behavior in the large deformation regime. Additionally, because of its intrinsic nonlinearity, the equations of nonlinear elasticity provide a rich basis for purely mathematical studies in, for example, nonlinear analysis. Thus, the subject is wide-ranging in both its theoretical and application perspectives, cf. [12].

Some of the publications from this area that are closest to our problem are listed below. Oden and Childs [13] studied the post-buckling problem of finite deflections of a clamped-free column subjected to an axial force and constructed of a nonlinearly elastic material characterized by a moment-curvature law similar to that exhibited by a class of elastoplastic materials. Lewis and Monasa [14] solved the problem of large deflections of a prismatic cantilever beam made of Ludwick type materials subjected to an end moment. The results are given for the vertical and horizontal deflections at the free end of the beam for rotations less than $\pi / 2$. Kounadis and Mallis [15] dealt with the post-buckling response of a simply supported,

* Corresponding author, e-mail: miha.brojan@fs.uni-lj.si, Phone: +38614771604, Fax: +38612518567 


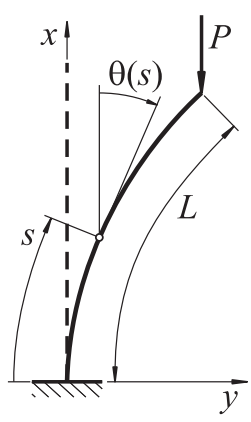

Fig. 1 The buckled column.

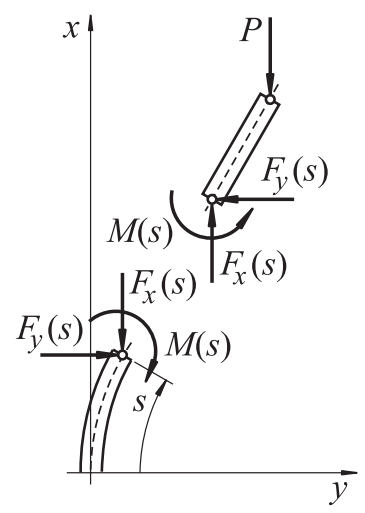

Fig. 2 Free body diagram of a deflected column.

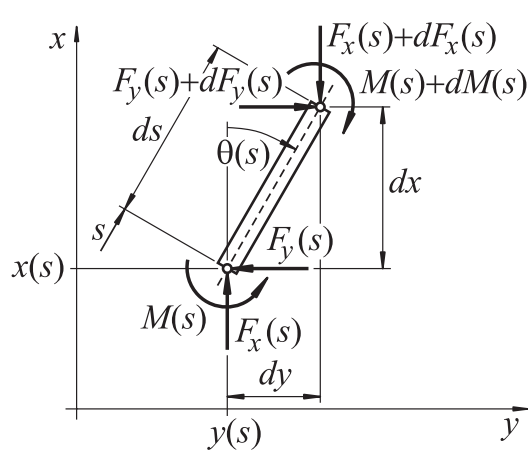

Fig. 3 Infinitesimal element of the deflected column.

axially compressed, uniform bar of nonlinearly elastic material. Wang [16] investigated the nonlinear buckling of an elastic column with softening characteristic. He found that, under large disturbances, the column may buckle under loads well below the linear buckling load. Jung and Kang [17] analyzed buckling of a prismatic, inextensible column fiber which is considered to be with no shear effect and whose constitutive equation corresponds to a Ludwick or modified Ludwick type. They presented solutions for four different combinations of a horizontal and vertical direction of point and distributed load.

The aim of this article is to investigate critical and post-critical behavior of an inextensible nonlinearly elastic column made of Ludwick type material and subjected to a compressive force.

\section{Formulation of the problem}

The mathematical model of the discussed problem is based on elastica theory, assuming that:

- the column is incompressible;

- shear stresses are negligible in comparison with the normal stresses when the length-to-height ratio of the column is large;

- Bernoulli hypothesis, which states that plane cross-sections, which are perpendicular to the neutral axis before deformation, remain plain and perpendicular to the neutral axis after deformation and do not change their shape and area.

It then follows that the Euler-Bernoulli equation, which states that local bending moment is solely proportional to the local curvature, is valid.

Consider a slender, initially straight homogenous column of length $L$ subjected to an end axial compressive force $P$, as shown in Fig. 1. The Cartesian $(x, y)$-coordinate system is chosen in such a manner that the ordinate axis coincides with the axis of the undeformed column and the coordinate origin is located at its clamped end.

Let $s, 0 \leq s \leq L$, be a curvilinear coordinate along the axial line measured from the clamped end and $\theta(s)$ the angle between the positive $x$-axis and the tangent to the neutral axis at point $s$. The column has a rectangular cross-section of constant height $h$ and constant width $a$.

\section{The governing equations}

The concepts and assumptions stated in previous sections will serve as a starting point for the derivation of governing equations. Consider the upper segment of the deflected column as a free body diagram as shown in Fig. 2.

Equations of horizontal and vertical equilibrium of forces respectively yield

$$
\begin{aligned}
& F_{x}(s)=P, \\
& F_{y}(s)=0 .
\end{aligned}
$$

In Fig. 3 the infinitesimal element of the deflected column is given. It follows from equilibrium of forces that $d F_{x}(s)=0$ and $d F_{y}(s)=0$.

Thus, the equilibrium of moments on the infinitesimal element Eqs. (1) and (2) yields

$$
d M+P d y=0 .
$$




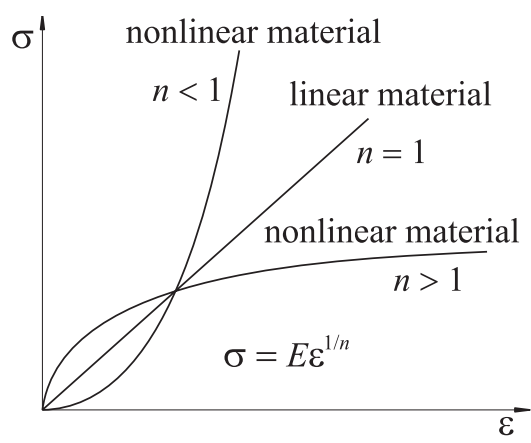

Fig. 4 Stress-strain relation for Ludwick type material in tensile domain.

Using geometrical relations

$$
\frac{d y}{d s}=\sin \theta \text { and } \frac{d x}{d s}=\cos \theta
$$

Eq. (3) takes the form

$$
\frac{d M}{d s}+P \sin \theta=0
$$

As mentioned previously, the column is made of nonlinearly elastic material for which the experimental stress-strain relation is represented by the Ludwick constitutive law, i.e.

$$
\sigma:=\left\{\begin{aligned}
E \cdot|\varepsilon|^{1 / n} ; & \varepsilon \geq 0 \\
-E \cdot|\varepsilon|^{1 / n} ; & \varepsilon<0
\end{aligned}\right.
$$

where $\sigma$ and $\varepsilon$ are the stress and strain (for tensile $\varepsilon \geq 0$ and compressive $\varepsilon<0$ domain), respectively, and $E, n$ are material constants, cf. Fig. 4. Note that expression (6) has one shortcoming - the stress gradient goes to infinity for $n>1$ (and to zero for $n<1$ ) when the strain value reaches zero. To overcome this problem Jung and Kang suggested a modified form of Eq. (6), cf. Eq. (2) in [17]. However, the non-modified expression (6) is used in our calculations for the sake of clarity of the derived equations. Nevertheless, the modified model (or any other similar model, for that matter) could be used with only minor adjustments and considerably more numerical work.

It is known that the inner bending moment, acting at any cross-section of the column, can be expressed with normal stress $\sigma$, as follows

$$
M=-\int_{A} \sigma y d A,
$$

where $\sigma$ is related to corresponding strain in tension and compression, cf. Eq. (6). Using the normal strain-curvature expression $\varepsilon=y \rho^{-1}$, we find

$$
M=E I_{n} \frac{1}{\rho^{1 / n}},
$$

where

$$
I_{n}=\frac{1}{2^{(1+n) / n}} \frac{n}{2 n+1} h^{(1+2 n) / n} a
$$

has been introduced. Taking into account the expression for curvature

$$
\frac{1}{\rho}=\frac{d \theta}{d s},
$$

Eq. (8) thus become

$$
M=E I_{n}\left(\frac{d \theta}{d s}\right)^{1 / n} .
$$


Differentiating Eq. (11) with respect to $s$ and combining it with Eq. (5) one can derive

$$
E I_{n} \frac{1}{n}\left(\frac{d \theta}{d s}\right)^{(1-n) / n} \frac{d^{2} \theta}{d s^{2}}+P \sin \theta=0 .
$$

It can be noted that Eq. (12), together with boundary conditions

$$
\begin{aligned}
\theta(s=0) & =0, \\
\frac{d \theta}{d s}(s=L) & =0,
\end{aligned}
$$

forms the set of equations for solving the problem of an axially loaded column. Furthermore, by introducing the quantities

$$
\tilde{s}:=\frac{s}{L}, \quad \tilde{x}:=\frac{x}{L}, \quad \tilde{y}:=\frac{y}{L}, \quad \text { and } \quad \tilde{P}:=\frac{n L^{(1+n) / n} P}{E I_{n}},
$$

where all lengths were normalized with $L$ and force by $E I_{n} /\left(n L^{(1+n) / n}\right)$, Eqs. (12)-(14) can be written in dimensionless form

$$
\begin{aligned}
\left(\frac{d \theta}{d \tilde{s}}\right)^{(1-n) / n} \frac{d^{2} \theta}{d \tilde{s}^{2}}+\tilde{P} \sin \theta & =0, \\
\theta(\tilde{s}=0) & =0, \\
\frac{d \theta}{d \tilde{s}}(\tilde{s}=1) & =0,
\end{aligned}
$$

which is more suitable for further treatment.

\section{Analytical determination of the critical force}

At immediate post-buckling $\theta(\tilde{s})$ is expected to be small for all $\tilde{s}, 0 \leq \tilde{s} \leq 1$. Therefore approximating $\sin \theta \approx \theta$ is reasonable. Introducing variable $U$,

$$
\frac{d \theta}{d \tilde{s}}=: U(\theta)
$$

and differentiating with respect to $\tilde{s}$ gives

$$
\frac{d^{2} \theta}{d \tilde{s}^{2}}=U^{\prime} U
$$

Eq. (16) can now be rewritten as

$$
U^{1 / n} U^{\prime}+\tilde{P} \theta=0 .
$$

Integrating Eq. (21)

$$
\frac{n}{1+n} U^{(1+n) / n}+\frac{\tilde{P}}{2} \theta^{2}=C,
$$

and using Eq. (19) yield

$$
\frac{n}{1+n}\left(\frac{d \theta}{d \tilde{s}}\right)^{(1+n) / n}+\frac{\tilde{P}}{2} \theta^{2}=C .
$$

The boundary condition (18) gives

$$
C=\frac{\tilde{P}}{2} \theta_{e}^{2}
$$


where $\theta_{e}:=\theta(\tilde{s}=1)$. Thus

$$
\frac{d \theta}{d \tilde{s}}=\left(\frac{1+n}{n}\right)^{n /(1+n)}\left(\frac{\tilde{P}}{2}\right)^{n /(1+n)}\left(\theta_{e}^{2}-\theta^{2}\right)^{n /(1+n)} .
$$

Integrating once more

$$
\int_{y(\tilde{s}=0)}^{y(\tilde{s}=1)} \frac{d \theta}{\left(\theta_{e}^{2}-\theta^{2}\right)^{n /(1+n)}}=\left(\frac{1+n}{n}\right)^{n /(1+n)}\left(\frac{\tilde{P}}{2}\right)^{n /(1+n)},
$$

and approximating $\theta(\tilde{s})$ with the linear function in $\tilde{s}$, i.e. $\theta:=\theta_{e} \tilde{s}$, one can get

$$
\int_{0}^{1} \frac{\theta_{e} d \tilde{s}}{\theta_{e}^{2 n /(1+n)}\left(1-\tilde{s}^{2}\right)^{n /(1+n)}}=\left(\frac{n+1}{n}\right)^{n /(1+n)}\left(\frac{\tilde{P}}{2}\right)^{n /(1+n)} .
$$

The integral on the left side can be expressed with the Beta function,

$$
\mathrm{B}(p, q)=\int_{0}^{1} t^{p-1}(1-t)^{q-1} d t
$$

which leads to

$$
\frac{1}{2} \theta_{e}^{(1-n) /(1+n)} \mathrm{B}\left(\frac{1}{2}, \frac{1}{1+n}\right)=\left(\frac{1+n}{n}\right)^{n /(1+n)}\left(\frac{\tilde{P}}{2}\right)^{n /(1+n)} .
$$

Thus the force $\tilde{P}$ at immediate post-buckling is

$$
\tilde{P}=\frac{n}{1+n} 2^{-1 / n} \mathrm{~B}\left(\frac{1}{2}, \frac{1}{1+n}\right)^{(1+n) / n} \theta_{e}^{(1-n) / n} .
$$

The critical force for linearly elastic material can be obtained by letting $\theta_{e}$ be very small and $n=1$.

$$
\tilde{P}_{c r}=\frac{1}{4} \mathrm{~B}\left(\frac{1}{2}, \frac{1}{2}\right)^{2}=\frac{\pi^{2}}{4} .
$$

This value is well known and is identical to that found in literature $[9,11]$.

\section{Numerical integration}

Based on the above analysis, a computer program was developed to solve differential Eqs.(16)-(18). The Runge-KuttaFehlberg and bisection methods are used to find the solution. But first, parameter $t$ is introduced

$$
t:=\tilde{P}^{n /(1+n)} \tilde{s} .
$$

The governing equation (16), and boundary conditions (17) and (18) now take the form

$$
\begin{aligned}
\left(\frac{d \theta}{d t}\right)^{(1-n) / n} \frac{d^{2} \theta}{d t^{2}}+\sin \theta & =0, \\
\theta(t=0) & =0, \\
\frac{d \theta}{d t}(t=t *) & =0 .
\end{aligned}
$$

The algorithm for the solution of the discussed problem is summarized as follows: 
Step 1: $\quad$ Value of $\mu_{0}$ is fixed, where

$$
\mu_{0}:=\frac{d \theta}{d t}(t=0)
$$

Step 2: Eqs. (33), (34), and (36) are integrated as initial value problem by the Runge-Kutta-Fehlberg algorithm.

Step 3: $\quad$ Bisection method is employed to find the value of $t *$ that satisfies Eq. (35).

Step 4: The normalized compressive force and moment at the clamped end are computed from Eqs. (32) and (36), i.e.

$$
\begin{aligned}
\tilde{P} & =(t *)^{(1+n) / n}, \\
\frac{d \theta}{d \tilde{s}}(\tilde{s}=0) & =t * \mu_{0} .
\end{aligned}
$$

Step 5: Having obtained these values, Eqs. (16), (17), (38), normalized geometrical relations (4), i.e.

$$
\frac{d \tilde{y}}{d \tilde{s}}=\sin \theta \text { and } \frac{d \tilde{x}}{d \tilde{s}}=\cos \theta
$$

together with boundary conditions

$$
\tilde{y}(\tilde{s}=0)=0 \text { and } \tilde{x}(\tilde{s}=0)=0
$$

can now be integrated in terms of the variable $\tilde{s}$ for the shape of the column in the Cartesian $(\tilde{x}, \tilde{y})$-coordinate system without difficulty.

The accuracy of the solution is estimated by comparing the results to the elliptic integral formulation. After introducing parameter

$$
\theta_{e}:=\theta(t=t *)
$$

taking into account Eqs. (33), (35) we can, after some work, get $t *$ as follows

$$
t *=\left(\frac{1+n}{n}\right)^{-n /(1+n)} \int_{0}^{\theta_{e}} \frac{d \theta}{\left(\cos \theta-\cos \theta_{e}\right)^{n /(1+n)}} .
$$

The normalized moment at the clamped end is

$$
\frac{d \theta}{d \tilde{s}}(\tilde{s}=0)=t *\left(\frac{1+n}{n}\right)^{n /(1+n)}\left(1-\cos \theta_{e}\right)^{n /(1+n)} .
$$

Normalized force $\tilde{P}$ is calculated in the same way as before, see Eq. (37). Note that employing Eqs. (16), (18), (41), (32), and (39) results in

$$
\begin{aligned}
& \tilde{y}(\tilde{s})=\left(\frac{1+n}{n} \tilde{P}\right)^{-n /(1+n)} \int_{0}^{\theta(\tilde{s})} \frac{\sin \theta d \theta}{\left(\cos \theta-\cos \theta_{e}\right)^{n /(1+n)}}, \\
& \tilde{x}(\tilde{s})=\left(\frac{1+n}{n} \tilde{P}\right)^{-n /(1+n)} \int_{0}^{\theta(\tilde{s})} \frac{\cos \theta d \theta}{\left(\cos \theta-\cos \theta_{e}\right)^{n /(1+n)}} .
\end{aligned}
$$

Integrals in Eqs. (42), (44), and (45) are solved using Gaussian quadrature formula. The results are discussed in the next section.

\section{Results and discussion}

An excellent agreement between both previously described numerical approaches is obtained. Diagrams representing a load as a function of downward displacement $\delta$, where $\delta=1-\tilde{x}(\tilde{s}=1)$, are depicted in Fig. 5. Different values of nonlinearity 


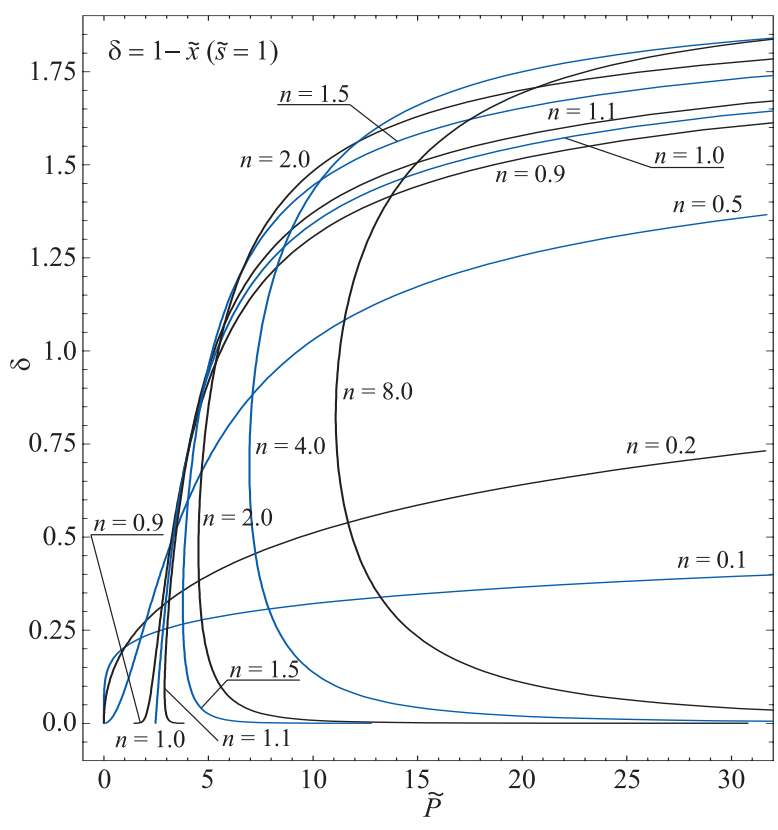

Fig. 5 (online colour at: www.zamm-journal.org) Load as a function of vertical displacement of the free end of the column.

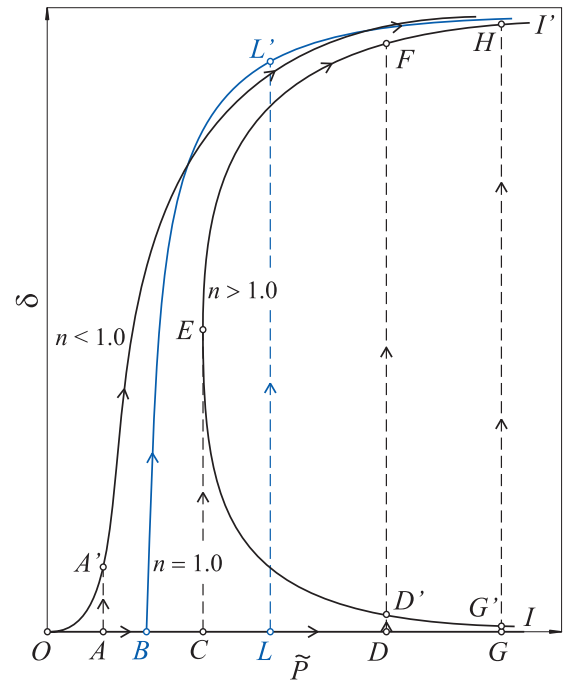

Fig. 6 (online colour at: www.zammjournal.org) Sketch of the $\tilde{P}-\delta$ curve.

\begin{tabular}{llllll}
\hline$n$ & 1.1 & 1.5 & 2.0 & 4.0 & 8.0 \\
\hline$\tilde{P}$ & 2.903 & 3.777 & 4.523 & 6.958 & 11.09 \\
$\delta$ & 0.089 & 0.312 & 0.459 & 0.693 & 0.825 \\
$\tilde{P}$ & 2.903 & 3.777 & 4.523 & 6.958 & 11.09 \\
$\theta_{e}$ & 0.595 & 1.094 & 1.297 & 1.496 & 1.553 \\
\hline
\end{tabular}

Table 1 Numerical data for the local minimum of the $\tilde{P}-\delta$ and $\tilde{P}-\theta_{\mathrm{e}}$ curves.

parameter $n, n=0.1,0.2,0.5,0.9,1.0,1.1,1.5,2.0,4.0$, and 8.0, are selected only to better understand the influence of $n$ on the buckling response of the column.

A more detailed description of the matter is outlined in Fig. 6, where all three possible cases, i.e. $n<1, n=1, n>1$, are treated.

In a nonlinear case $n<1$, the bifurcation point is found at $O$, i.e. $\tilde{P}_{c r}=\tilde{P}_{O}=0$. The bifurcation $\tilde{P}$ - $\delta$ curve is monotonic. Two equilibrium solutions are found for any $\tilde{P}>0$, one unstable (on branch $O-I$, which corresponds to a straight column configuration), and one stable solution (on branch $O-I^{\prime}$ ). The most interesting observation in this example is that the bifurcation point is found at the beginning of the loading. This means if an infinitesimal disturbance is applied the column bends laterally for an arbitrary small load $\tilde{P}$. The loading path is thus $O-A^{\prime}-I^{\prime}$ or $O-A-A^{\prime}-I^{\prime}$ if a disturbance is met not until point $A$ at which the column jumps from state $A$ to state $A^{\prime}$. In that case for $\tilde{P}_{O} \leq \tilde{P}<\tilde{P}_{A}$ the column remains straight. As $\tilde{P}$ is decreasing gradually, the column moves from state $I^{\prime}$ to state $O$, the unloading path $I^{\prime}-A^{\prime}-O$ is obtained. To conclude, in case $n<1$ it is observed that the column may buckle under loads well below the linear buckling load.

In a linear case $n=1$, the well known force-displacement curve is found. A neutral equilibrium is maintained as load $\tilde{P}$ gradually increases from point $O$ to point $B$, where bifurcation occurs. The critical load is $\tilde{P}_{c r}=\tilde{P}_{B}=\pi^{2} / 4$. The bifurcation $\tilde{P}-\delta$ curve is monotonic. Two equilibrium solutions are found in the post-buckling domain, one is stable, (on $B-I^{\prime}$ branch), and one is unstable (on $B-I$ branch which corresponds to a straight column configuration), as already known. Things are similar as in the case $n<1$. The loading-unloading paths are $O-B-L^{\prime}-I^{\prime}\left(O-L-L^{\prime}-I^{\prime}\right)$ and $I^{\prime}-L^{\prime}-B-O$, respectively.

The most important result in the present paper refers to the analysis of the nonlinear case $n>1$. Figs. 5 and 6 show that the characteristic $\tilde{P}-\delta$ curve $(n>1)$ is not monotonic (no bifurcation points are found). As $\tilde{P}$ increases, $\delta$ is decreasing for the $I-D^{\prime}-E$ segment, but increasing for the $E-F-I^{\prime}$ segment of the path. Point $E$ is the local minimum of the $\tilde{P}-\delta$ curve. Numerical data are given in Table 1.

It is known that the area under the curve represents the work done on the elastica, therefore a negative slope signifies negative work for a positive displacement. Thus the equilibrium states on the $I-D^{\prime}-E$ branch are statically unstable for a given constant load. For $\tilde{P}<\tilde{P}_{C}$ the only solution is the stable straight column; for $\tilde{P}=\tilde{P}_{C}$ there are two solutions, one stable solution at point $E$ and one at point $C$ which corresponds to a straight column. The equilibrium state at point $C$ is statically 


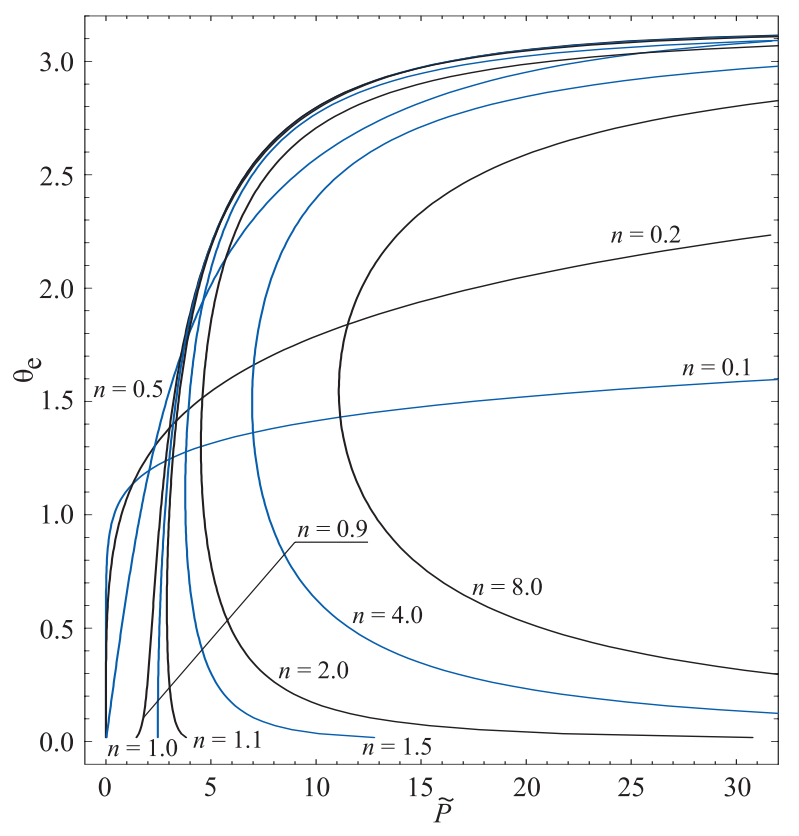

Fig. 7 (online colour at: www.zamm-journal.org) Load as a function of inclination of the free end of the column.

unstable, since sufficient finite disturbance may cause the column to jump between states $C$ and $E$; for $\tilde{P}_{C}<\tilde{P} \leq \tilde{P}_{D}$ there are three solutions, one stable and two unstable solutions. The unstable equilibrium states can be found on the $C$ - $D$ (straight column) and $D^{\prime}-E$ segments, whereas the stable states are met on the $E-F-I^{\prime}$ segment of the path. It should be noted point $D$ is defined in such a manner that a finite disturbance needed for column to snap between equilibrium states at point $D$ is significantly smaller than that at point $C$; for $\tilde{P}_{D} \ll \tilde{P}, \tilde{P} \rightarrow \infty$, two solutions are found, one unstable solution at point $I$, which is a straight column configuration, and one stable at point $I^{\prime}$. In this case an infinitesimal disturbance is sufficient for the column to buckle, since points in a relation such as $G$ and $G^{\prime}$ coincide as $\tilde{P} \rightarrow \infty$, i.e. branches $G^{\prime}-I$ and $G-I$ asymptotically approach each other as $\tilde{P} \rightarrow \infty$. Note that the loading-unloading paths differ. Namely, if an initially straight column is loaded with $\tilde{P}$, the column remains straight until point $C$ is met. A further increase in force $\tilde{P}$ may result in a violent jump between states, e.g. from state $D$ to state $F$, if a sufficient finite disturbance is applied (or from state $G$ to state $H$ in case of an infinitesimal disturbance), followed by branch $H-I^{\prime}$. The unloading path is obtained if $\tilde{P}$ is gradually decreased. The column goes through the states $I-H-F-E$. A further decrease in $\tilde{P}$ results in a snap back to state $C$ which is a straight column configuration. Point $C$ is followed by $B-O$ branch. A hysteresis loop thus exists.

In paper [16] Wang analyzed an example that corresponds to our case $n>1$. He used nonlinear constitutive equation in the form of a moment-curvature relation which was formed in such a way that the column behaves as linearly elastic when deformations are small. This proves to be a significant distinction since it results in differences in determination of critical forces. Namely, in Wang's case all curves bifurcate from $\tilde{P}_{c r}=\tilde{P}_{B}=\pi^{2} / 4$ (bifurcation buckling is met) whereas in our case $\tilde{P}_{c r} \rightarrow \infty$ as $n$ is increased (disturbance buckling is met).

Similar conclusions can be made analyzing diagrams in Fig. 7. The figure shows curves representing load as a function of inclination of the free end of the column. Numerical data for the local minimum of the $\tilde{P}-\theta_{\mathrm{e}}$ curve are given in Table 1 .

Fig. 8 illustrates the comparison between approximative and numerical approach in $\tilde{P}-\theta_{\mathrm{e}}$ diagrams.

One can see that the approximative formula (30), developed for small $\theta_{e}$, is quite accurate even for $\theta_{e}$ as large as 1.2 for $n<1$ and $\theta_{e}$ as large as 0.7 for $n>1$, cf. Figs. 8a) and 8b). To illustrate the influence of the material constant $n$, Fig. 9 shows deflected shapes of linearly and nonlinearly elastic column for three different values $n$, at constant inclination of the column at the free end $\theta_{e}$. These diagrams when referred to Fig. 8 explain why the approximative formula is more accurate for $n<1$ than for $n>1$ or $n=1$. It can be seen from Fig. 9 that deformations of $n<1$ columns are significantly smaller than those of $n>1$ columns.

Practical examples of Ludwick type material are annealed copper and N.P.8 aluminum alloy, characterized by material constants $E=458.501 \mathrm{MPa}, n=0.463$, and $E=455.743 \mathrm{MPa}, n=0.209$, respectively, [14]. Columns made of these two materials thus exhibit the behavior of $n<1$ columns. 


\section{Conclusions}

The above nonlinear analysis discusses buckling and post-buckling response of linearly and nonlinearly elastic columns made of Ludwick type material. The solution of governing equations of buckling is obtained numerically. Variation of material constant $n(n<1, n=1, n>1)$ leads to different solutions. For $n<1$ a monotonic bifurcation curve is obtained. An interesting feature for this case is that the bifurcation point is found at the beginning of the loading which means that lateral bending of the column is possible even for arbitrary small axial loads if an infinitesimal disturbance is applied. An already known behavior of the linearly elastic column $(n=1)$ is confirmed by the nonlinear analysis presented in this paper. It is shown that the post-buckling behavior of $n>1$ columns exhibit various non-linear phenomena such as limit load and violent jumps similar to snap-through. Note that results $\tilde{P}_{c r}=0$ for $n<1$ and $\tilde{P}_{c r} \rightarrow \infty$ for $n>1$ are consequences of the shortcoming of the Ludwick constitutive formula, i.e. the stress gradient goes to infinity for $n>1$, and to zero for $n<1$, when the strain value reaches zero.

An analytical solution is also developed for nonlinearly elastic columns at immediate post-buckling, i.e. when $\theta(\tilde{s})$ is expected to be small for all $\tilde{s}, 0 \leq \tilde{s} \leq 1$. Good agreement between the numerical and analytical approaches have been confirmed for the inclination of the column at the free end $\theta_{e}$ as large as 1.2 for $n<1$ and $\theta_{e}$ as large as 0.7 for $n>1$. Moreover, the same analysis may be used for treating the problem of a general nonlinear relation between stress and strain of the form $\sigma=f(\varepsilon)$, where $f$ is an experimental stress-strain function characterizing the structural material.
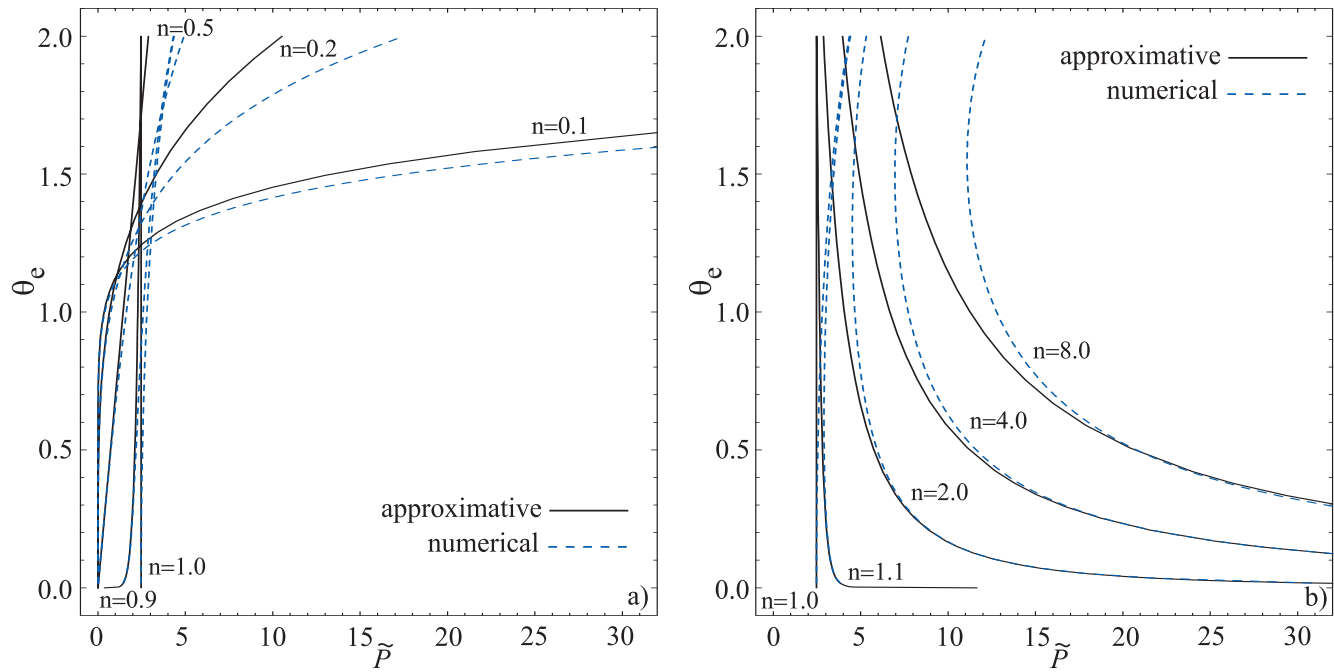

Fig. 8 (online colour at: www.zamm-journal.org) Comparison between approximative and numerical approach in $\tilde{P}-\theta_{\mathrm{e}}$ diagrams.

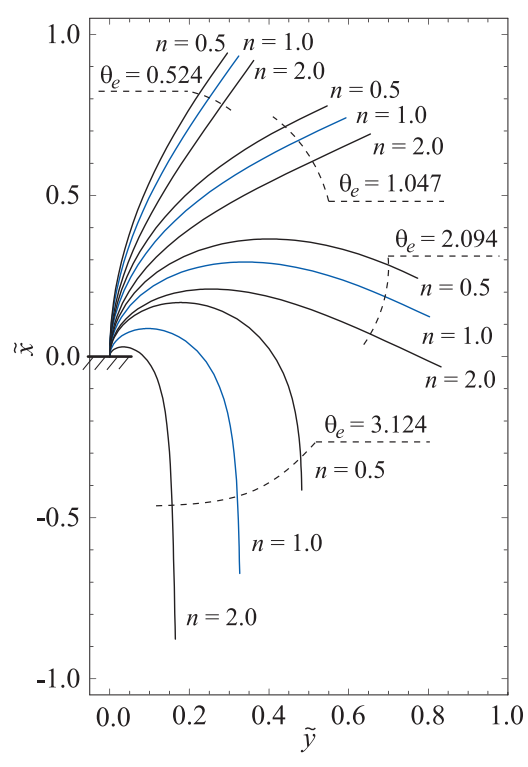

(c) 2007 WILEY-VCH Verlag GmbH \& Co. KGaA, Weinheim
Fig. 9 (online colour at: www.zamm-journal.org) Deflected shapes of the column. 


\section{References}

[1] V. Komkov, Z. Angew. Math. Mech. 67, 419-433 (1987).

[2] V.V. Kuznetsov and S. V. Levyakov, Prikl. Mekh. Tekh. Fiz. 40, 184-185 (1999).

[3] S.V. Levyakov, Prikl. Mekh. Tekh. Fiz. 42, 153-160 (2001).

[4] S. N. Korobeinikov, Secondary loss of stability of a simply supported rod, in: Proceedings of the 4th International Conference "Lavrent'ev Readings on Mathematics, Mechanics, and Physics", Novosibirsk, Russia, 1995, p. 104.

[5] B. Wu, Z. Angew. Math. Mech. 75, 741-751 (1995).

[6] C.Y. Wang, Int. J. Non-Linear Mech. 32, 115-1122 (1997).

[7] M.A. Vaz and D. F. C. Silva, Int. J. Non-Linear Mech. 38, 483-492 (2003).

[8] A.E. H. Love, A Treatise on the Mathematical Theory of Elasticity, 4th ed. (Dover Publications, New York, 1972).

[9] S.P. Timoshenko and J. M. Gere, Theory of Elastic Stability, 2nd ed. (McGraw-Hill, New York, 1961).

[10] S. S. Antman, Nonlinear Problems of Elasticity (Springer Verlag, Berlin, 1995).

[11] C. M. Wang, C.Y. Wang, and J. N. Reddy, Exact Solutions for Buckling of Structural Members (CRC Press, Boca Raton, FL, 2005).

[12] Y.B. Fu and R. W. Ogden, Nonlinear Elasticity: Theory and Applications (Cambridge University Press, Cambridge, 2001).

[13] J. T. Oden and S. B. Childs, J. Appl. Mech. 69, 48-52 (1970).

[14] G. Lewis and F. Monasa, Int. J. Nonlinear Mech. 17, 1-6 (1982).

[15] A. N. Kounadis and J. G. Mallis, Int. J. Non-Linear Mech. 22, 99-107 (1987).

[16] C.Y. Wang, Acta Mech. 119, 229-234 (1996).

[17] J.H. Jung and T. J. Kang, Textile Res. J. 75, 715-723 (2005). 